\title{
Narrow-Band Multiresonant Plasmon Nanostructure for the Coherent Control of Light: An Optical Analog of the Xylophone
}

\author{
Gaëtan Lévêque* and O. J.F. Martin ${ }^{\dagger}$ \\ Nanophotonics and Metrology Laboratory, Swiss Federal Institute of Technology Lausanne, \\ EPFL-STI-NAM ELG Station 11, CH-1015 Lausanne, Switzerland
}

(Received 12 November 2007; published 21 March 2008)

\begin{abstract}
We demonstrate that it is possible to combine several small metallic particles in a very compact geometry without loss of their individual modal properties by adding a gold metallic film underneath. This film essentially acts as a "ground plane" which channels the optical field of each particle and decreases the interparticle coupling. The localization of the electric field can then be controlled temporally by illuminating the chain with a chirped pulse. The sign of the chirp controls the excitation sequence of the particles with great flexibility.
\end{abstract}

DOI: 10.1103/PhysRevLett.100.117402

Some metallic subwavelength objects have the ability to enhance and localize the field of an incoming light at a specific wavelength corresponding to the excitation of a socalled localized surface-plasmon (LSP) mode [1,2]. It was recently shown that it is possible to control the near-field localization in time and/or space inside a complex metallic nanostructure using specifically designed light waves [35]. For example, varying the instantaneous frequency, the polarization, or the amplitude allows creating constructive interferences between the different modes of the nanostructure in order to confine light in well defined hot-spots. Early simulations used simple chirped pulses with a constant polarization. The creation of hot spots shortly localized in time (during a few ten femtoseconds) was evidenced this way, as well as apparent negative group velocity inside two-dimensional metallic tips [6,7]. The purpose of this Letter is to design a simple subwavelength metallic three dimensional structure which exhibits several LSP modes associated to nonoverlapping electric field distributions. The interest of such a structure is to create multiple addressable light spots whose excitation can be time-controlled using a custom designed chirped light pulse. Specifically, we show that simple parallelipipedic gold particles can be used as building blocks for that purpose, provided that the interparticle coupling efficiency is minimized in order to keep the field localized around mainly one particle at the LSP wavelength. The multiresonant character of the structure proposed here makes it particularly suitable for light control at the nanoscale. The first part of the Letter is devoted to the study of the spectroscopic properties of the proposed structure; the second part focuses on the interaction between its multiple LSP modes with a chirped femtosecond pulse to localize light in space and time.

The numerical simulations are performed with the Green's tensor method [8,9]. This method is adapted to the study of finite-size, two or three dimensional scatterers, embedded in a stratified background. It relies on the reso-
PACS numbers: 78.66.Bz, 71.45.Gm, 73.20.Mf, 78.20.Bh

lution of the Lippmann-Schwinger equation for the electric field. A great advantage of the Green's tensor method is that only the objects of interest need to be discretized. The boundary conditions at infinity are included in the Green's tensor of the stratified background. Details can be found in a recent review [10]. The gold permittivity data are from Palik [11].

The system consists in a chain of six gold particles aligned along the $y$-direction, Fig. 1 . The $y$ side has a constant length $a=50 \mathrm{~nm}$, while the $x$ side of each particle increases from $d=50 \mathrm{~nm}$ to $d=100 \mathrm{~nm}$ with steps of $10 \mathrm{~nm}$. The thickness is $20 \mathrm{~nm}$, and the spacing between each particle is $e=10 \mathrm{~nm}$. The entire system is illuminated from the silica substrate under total internal reflexion by an $s$-polarized plane wave (the incident electric field is parallel to the $x$ axis), which forms a $\theta=45^{\circ}$ angle with the normal to the interface. Each particle exhibits three LSP modes associated to each of its three sides (for the smallest particle, two of these modes are degenerate). In

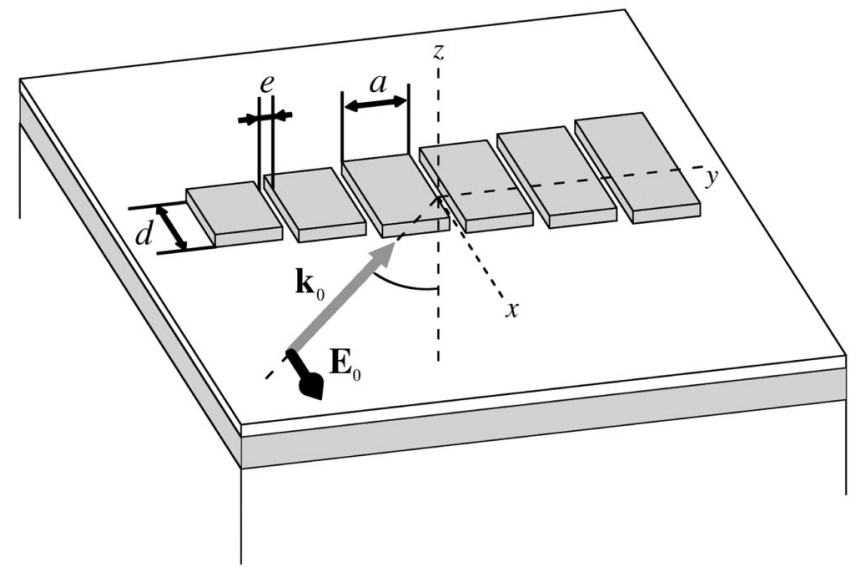

FIG. 1. Geometry of the system. The incident wave propagates parallel to the $y z$ plane; the incident electric field is parallel to the $x$ axis. Gray objects are made of gold; white objects are in silica $(\epsilon=2.25)$. 
this Letter, the incident wave is coupled to the mode corresponding to the longest side of each particle, whose resonance wavelength increases with the particle width $d$.

The optical response of this system strongly depends on the coupling between each adjacent particle. Figure 2(a) shows the absorption spectrum per volume unit for each particle incorporated in the whole chain lying on a semiinfinite silica substrate without gold layer. The vertical solid lines at the top of the figure indicate the position of the LSP resonance for each individual particle $(e=\infty)$. Two effects can be observed. The first one is due to the short length of the chain $(350 \mathrm{~nm})$, which makes that the excitation of any particle LSP is spread in the whole chain by nearest neighbor coupling. More precisely, it appears that the larger the particle, the higher its sensitivity to the

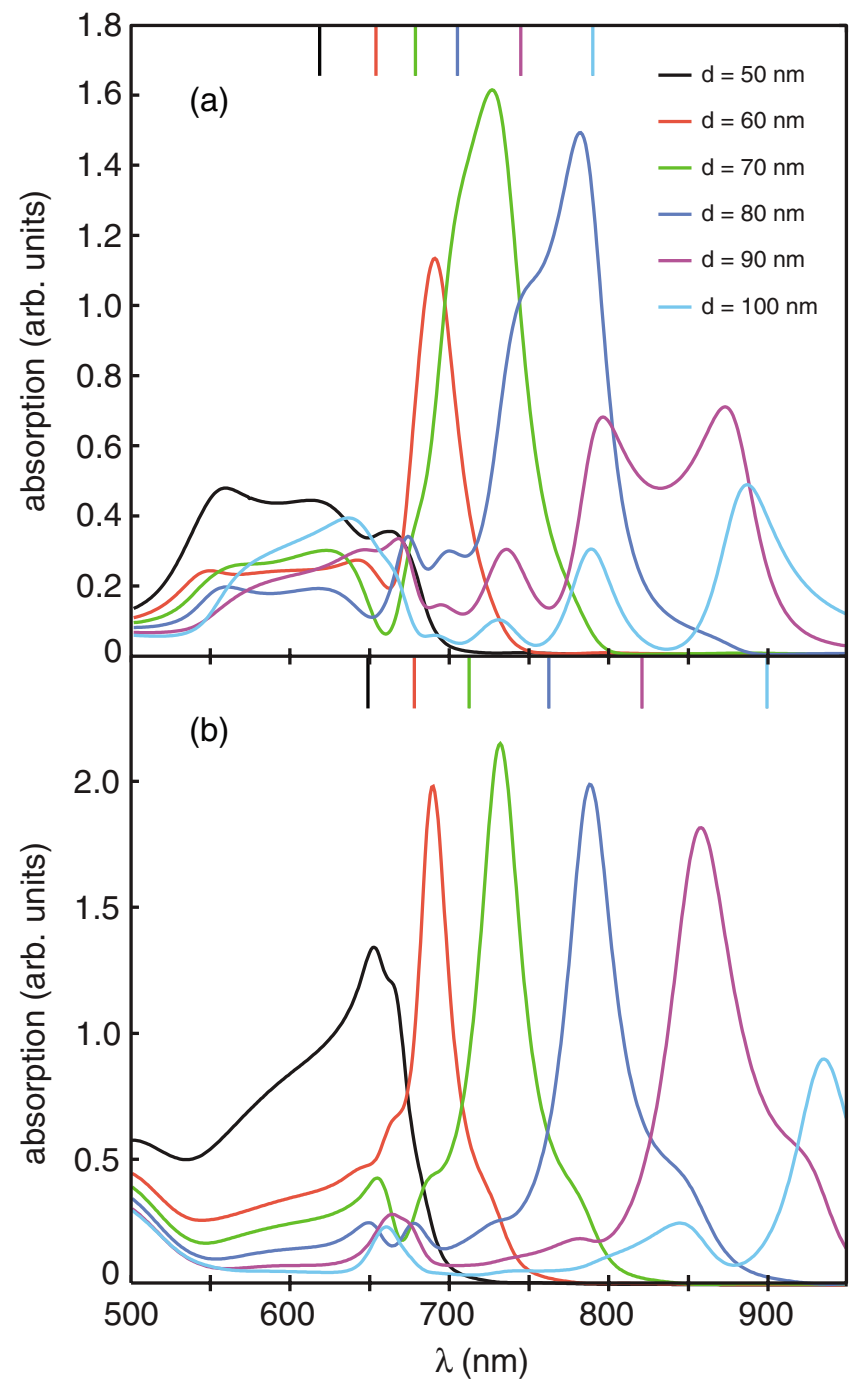

FIG. 2 (color online). Absorption spectrum computed in each particle of the system of Fig. 1 (a) without and (b) with the underneath gold film. The vertical solid lines at the top of each figure indicates the position of the LSP resonance of each isolated particle (a) without and (b) with the underneath gold film. other particles: the $d=60 \mathrm{~nm}$ particle has only one peak, whereas the $d=90 \mathrm{~nm}$ and the $d=100 \mathrm{~nm}$ particles present several secondary maxima. The larger the particle, the more it is sensitive to this effect since a larger facet intercepts more field than a smaller facet. This increases the reactivity of a large particle to the excitation of a smaller neighboring particle. This strong interparticle coupling can be modified by the presence of an underlying gold film as sketched in Fig. 1. This is visible in Fig. 2(b), where absorption spectra are shown for the geometry where the chain is separated from a $40 \mathrm{~nm}$-thick gold film by a $20 \mathrm{~nm}$-thick silica spacer. Let us emphasize that the delocalized LSP mode propagating on the gold film cannot be excited by the incident field, which is $s$-polarized [12]. The interaction of each individual particle with the film shifts its plasmon resonance to the red by about $30 \mathrm{~nm}$ (for the $d=50 \mathrm{~nm}$ particle) to $110 \mathrm{~nm}$ [for the $d=100 \mathrm{~nm}$ particle ; compare vertical lines in Figs. 2(a) and 2(b)]. Each particle in the chain presents now essentially one absorption peak, much narrower than the peak computed without the metallic film. This effect is particularly visible for the particles with side $d=50,80$ and $90 \mathrm{~nm}$. The origin of this phenomenon is the following: when the gold film is added, the field remains mainly concentrated between the particle and the gold film, which decreases the particle-particle coupling with respect to the film-particle coupling [13]. The smallest particle now resonates around $\lambda \approx 650 \mathrm{~nm}$, the largest one around $\lambda \approx$ $950 \mathrm{~nm}$. As in the previous case, the interaction between each particle induces an additional redshift, less important than that observed without a film: for particles of size $d=$ $60,70,80$, and $90 \mathrm{~nm}$, this shift is, respectively, 11, 23, 27, and $38 \mathrm{~nm}$. Hence, the system now exhibits six well defined resonances, whose wavelength increases regularly with the particle width. This relation between the frequency and the localization of light can be used to coherently control the optical near-field around the structure.

Indeed, by tuning the phase delay between the spectral components of an incoming pulse, it is possible to excite successively each particle in a specific sequence. In order to demonstrate this, the chain is illuminated with a light pulse whose large spectrum covers the six resonances of the system. The same incidence angle and polarization as previously are used. The pulse is synthesized by a sum of planewaves, which are sampled every nanometer. The amplitude and phase of each spectral component has been chosen in order to produce a pulse of homogeneous amplitude and decreasing instantaneous wavelength on a time of $200 \mathrm{fs,} \mathrm{Fig.} \mathrm{3.} \mathrm{The} \mathrm{wavelength} \mathrm{evolves} \mathrm{from}$ $1.0 \mu \mathrm{m}$ at $t=-100$ fs to $570.0 \mathrm{~nm}$ at $t=100 \mathrm{fs}$.

Maps of the electric field amplitude just above the chain as a function of time are shown in Fig. 4. The field was computed $30 \mathrm{~nm}$ above the vacuum-silica interface. When the pulse impinges on the metallic system, the LSP resonance of each particle is successively excited. Because of 


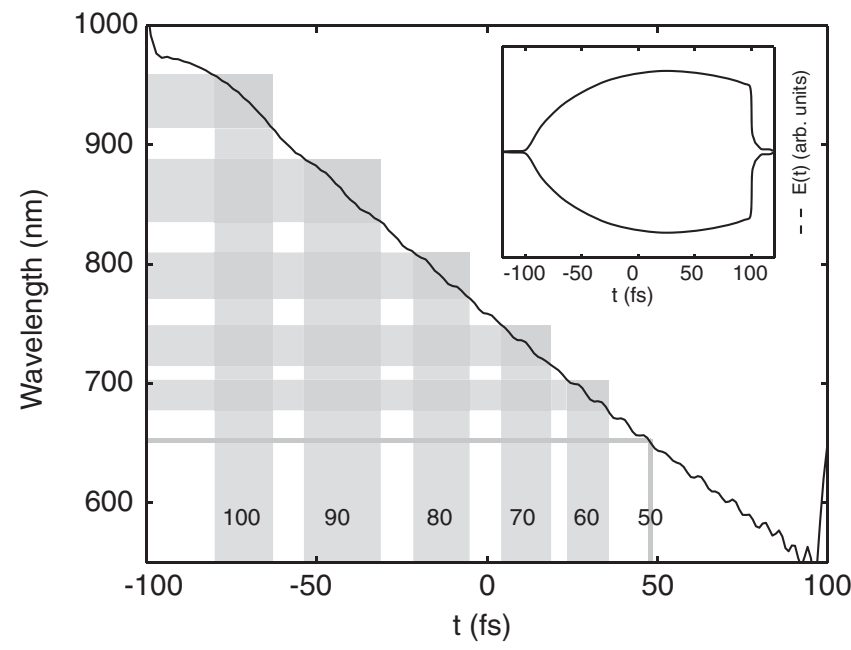

FIG. 3. Time evolution of the instantaneous wavelength during the pulse, at $x, y, z=0$. The gray horizontal bands represent the width of each peak for the particles $d=60 \mathrm{~nm}$ to $d=100 \mathrm{~nm}$ (see numbers inside the gray areas). The gray vertical bands represent the corresponding time width of the effective pulse seen by each particle. For the $d=50 \mathrm{~nm}$ particle, only the position of the resonance and the corresponding time have been reported. Inset: envelope $E(t)$ of the pulse amplitude, at $x, y, z=0$.

the decaying wavelength, the particle placed further away from the light source is excited first; then each particle from the widest to the narrowest follows: the energy seems to propagate backward, Fig. 4. The field is remarkably confined within each particle during the pulse and does not extend further away than the particle immediately shorter than the excited one, which demonstrates the good spatial localization of the electric field with time. Moreover, the amplitude of the field inside each excited particle follows the amplitude of the incident pulse.

Figure 5 shows the average amplitude $E_{p}$ of the electric field inside each particle of the chain as a function of time. The full width at half maximum of $E_{p}$ inside each particle is a few ten femtoseconds and decreases with the side from 50 fs for the $d=90 \mathrm{~nm}$ particle down to $25 \mathrm{fs}$ for the $d=$ $50 \mathrm{~nm}$ particle. The field in the largest particle has roughly constant value during the entire pulse duration. This figure shows that the time scale of the pulse seen by each of the five smallest particles is much shorter than the whole pulse duration: the light is temporally confined by the coupling to LSP. Two sets of three movies can be found in the auxiliary materials [14]. The first set (labeled a) and the second set (labeled b) correspond, respectively, to the system without and with the gold film. They show the evolution of the mean field amplitude inside the chain as a function of time for three different pulses. The first is the previously described pulse (the movie is simply a full time sequence corresponding to Fig. 5), the second is the exactly reversed pulse where the wavelength increases with time, and the last one has an increasing wavelength from -100 fs to $0 \mathrm{fs}$
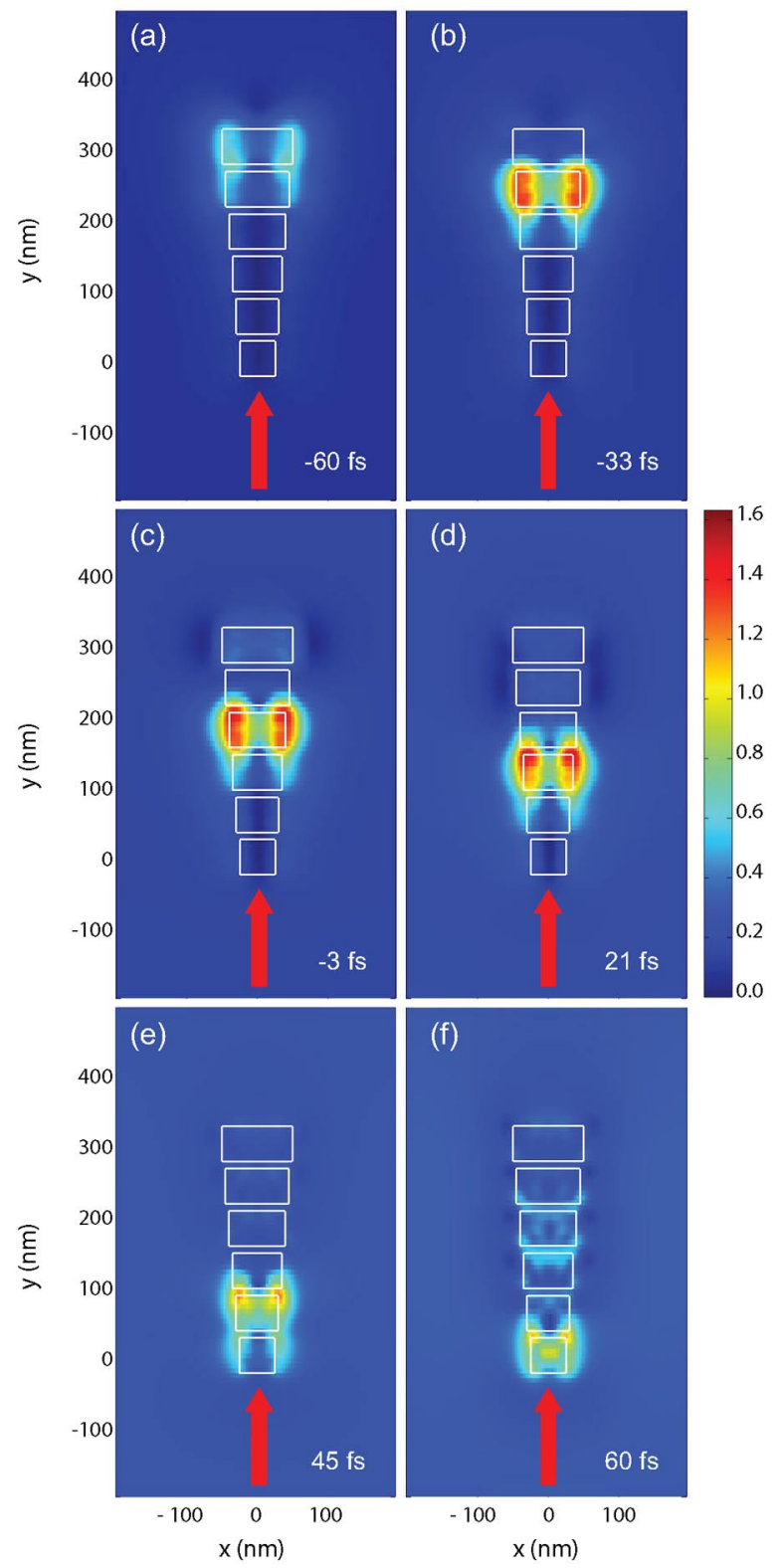

FIG. 4 (color online). Amplitude of the electric field normalized to the maximum amplitude of the incident field at the different times corresponding to the maximum of field amplitude above each particle. The field was computed $30 \mathrm{~nm}$ above the silica substrate.

followed by a decreasing wavelength from 0 to $100 \mathrm{fs}$. The result is a field maximum going forth and back along the chain. The amelioration of the field localization and propagation due to the gold film appears clearly. These movies emphasize the high degree of control that can be achieved for the localization in space and time of the LSP inside the chain.

Another interesting point is the relation between the time for which $E_{p}$ reaches its maximum inside one particular particle and the time at which the instantaneous wavelength of the incident pulse matches its LSP wave- 


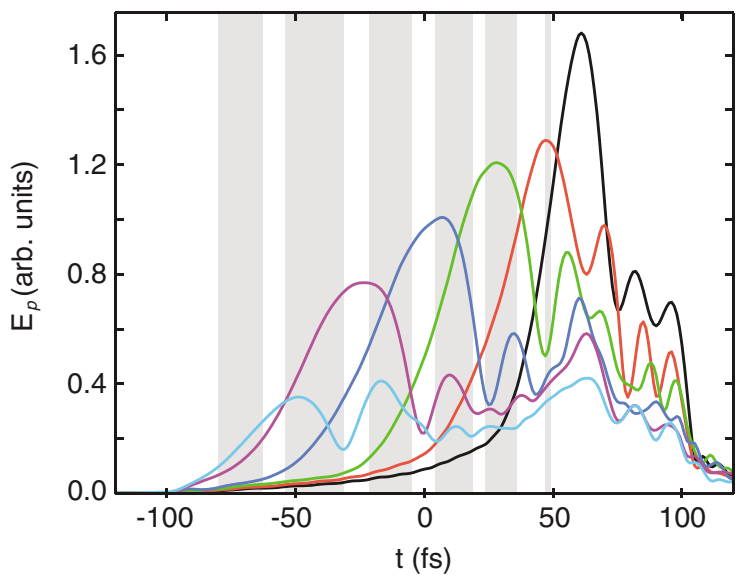

FIG. 5 (color online). Average electric field amplitude $E_{p}$ inside each particle of the chain, as a function of time. The vertical gray areas are the same as in Fig. 3.

length computed from absorption spectra [Fig. 2(b)]. In Fig. 3, the horizontal gray bands represent the width at half maximum for the absorption peaks in Fig. 2(b). This wavelength interval reported on the horizontal axis defines a time interval (vertical gray bands) during which the instantaneous wavelength matches the plasmon mode of each particle. The larger time interval ( $22 \mathrm{fs}$ ) is obtained for the $d=90 \mathrm{~nm}$ particle, and decreases with the width of the particle down to $13 \mathrm{fs}$ for the $d=60 \mathrm{~nm}$ particle. As the absorption peak of the $d=50 \mathrm{~nm}$ particle is very wide due to interband transitions in gold at short wavelengths, the width of the plasmon mode cannot be defined, and only the position of the maximum and the corresponding time have been plotted. These time intervals have been reported in Fig. 5. It appears that, for all particles, the time of maximal $E_{p}$ lies outside the corresponding window. This fact shows a clear delay between the time for which the incident wavelength corresponds to the LSP wavelength and the response of the particle. Moreover, by evaluating from Figs. 2(b) and 3 the precise time at which $\lambda(t)=$ $\lambda_{\mathrm{LSP}}$, we find an average delay of about 10-20 fs with the $E_{p}$ peak time in each particle. The reason for this delay is not clearly understood, but further calculations showed that a strong correlation exists with the coherence time of the LSP mode, deduced from the full width at half maximum of each absorption peak in Fig. 2(b), which is also about 10 to $20 \mathrm{fs}$. This delay indicates the time that a particle needs to adapt to the rapid change of frequency it experiences.

As a conclusion, we have shown in this Letter that it is possible to combine several small metallic particles in a very compact geometry without loss of their individual modal properties by adding a gold metallic film underneath. This film essentially acts as a "ground plane" which channels the optical field of each particle and decreases the interparticle coupling. The localization of the electric field can hence be controlled temporally by illuminating the chain with a chirped pulse. The sign of the chirp controls the excitation sequence of the particles with great flexibility. Finally, a comparison between the time at which the field is maximum in one particle and the time for which the wavelength of the incident chirped pulse corresponds to the LSP wavelength of the same particle indicates that the particles need a delay of few tens fentoseconds to adapt to the rapid change of frequency it experiences. This delay is correlated to the LSP mode coherence time.

Funding from the IST Network of Excellence Plasmonanodevices (No. FP6-2002-IST-1-507879) and from the Swiss National Science Foundation (R'Equip No. 206021) are gratefully acknowledged.

*gaetan.leveque@tyndall.ie

Tyndall National Institute, Lee Maltings, Prospect Row, Cork, Ireland.

${ }^{\dagger}$ http://nam.epfl.ch/

[1] H. Raether, Plasmons on Smooth and Rough Surfaces and on Gratings (Springer-Verlag, Berlin, 1988).

[2] J. P. Kottmann, O. J. F. Martin, D. R. Smith, and S. Schultz, Phys. Rev. B 64, 235402 (2001).

[3] M. Sukharev and T. Seideman, Nano Lett. 6, 715 (2006).

[4] M. Aeschlimann and M. Bauer et al., Nature (London) 446, 301 (2007).

[5] T. Brixner and F. J. Garcia de Abajo et al., Phys. Rev. B 73, 125437 (2006).

[6] T.-W. Lee and S. K. Gray, Phys. Rev. B 71, 035423 (2005).

[7] M. I. Stockman, S. V. Faleev, and D. J. Bergman, Phys. Rev. Lett. 88, 067402 (2002).

[8] O.J.F. Martin and N. B. Piller, Phys. Rev. E 58, 3909 (1998).

[9] M. Paulus and O. J. F. Martin, J. Opt. Soc. Am. A 18, 854 (2001).

[10] C. Girard, Rep. Prog. Phys. 68, 1883 (2005).

[11] E. D. Palik, Handbook of Optical Constants of Solids (Academic Press, New York, 1985).

[12] G. Lévêque and O. J. F. Martin, Opt. Lett. 31, 2750 (2006).

[13] G. Lévêque and O.J.F. Martin, Opt. Express 14, 9971 (2006).

[14] See EPAPS Document No. E-PRLTAO-100-090812 for movies showing the dynamics of the system. For more information on EPAPS, see http://www.aip.org/pubservs/ epaps.html. 